\title{
2 Reciprocal regulation of Aurora kinase A and ATIP3 in the control of metaphase spindle length
}

\author{
4 Anne Nehlig ${ }^{1,2,3} \cdot$ Cynthia Seiler $^{1,2,3} \cdot$ Yulia Steblyanko $^{4} \cdot$ Florent Dingli $^{5}$ - Guillaume Arras $^{5} \cdot$ Damarys Loew $^{5}$. \\ 5 Julie Welburn $^{6} \cdot$ Claude Prigent $^{7} \cdot$ Marin Barisic $^{4,8} \cdot$ Clara Nahmias $^{1,2,3}$ (D)
}

Received: 30 January 2020 / Revised: 18 July 2020 / Accepted: 7 August 2020

(c) Springer Nature Switzerland AG 2020

\section{Abstract}

Maintaining the integrity of the mitotic spindle in metaphase is essential to ensure normal cell division. We show here that depletion of microtubule-associated protein ATIP3 reduces metaphase spindle length. Mass spectrometry analyses identi-AQ2 fied the microtubule minus-end depolymerizing kinesin Kif2A as an ATIP3 binding protein. We show that ATIP3 controls metaphase spindle length by interacting with Kif2A and its partner Dda3 in an Aurora kinase A-dependent manner. In the absence of ATIP3, Kif2A and Dda3 accumulate at spindle poles, which is consistent with reduced poleward microtubule flux and shortening of the spindle. ATIP3 silencing also limits Aurora A localization to the poles. Transfection of GFP-Aurora A, but not kinase-dead mutant, rescues the phenotype, indicating that ATIP3 maintains Aurora A activity on the poles to control Kif2A targeting and spindle size. Collectively, these data emphasize the pivotal role of Aurora kinase A and its mutual regulation with ATIP3 in controlling spindle length.

\section{Keywords Aurora A · Dda3 $\cdot$ Kif2A $\cdot$ MTUS1 $\cdot$ Mitotic kinase $\cdot$ Mitotic spindle $\cdot$ Poleward microtubule flux}

\section{Introduction}

The size of the mitotic spindle in metaphase varies among different cell types and species but remains constant for a given cell type, despite high dynamics of spindle microtubules [1-3]. The spindle in metaphase must reach an optimal size to capture and bi-orient all duplicated chromosomes prior to cell division, and to allow separation of

Electronic supplementary material The online version of this article (https://doi.org/10.1007/s00018-020-03614-8) contains supplementary material, which is available to authorized users.

Clara Nahmias

clara.nahmias@inserm.fr

1 Inserm U981, Department of Molecular Medicine, Gustave Roussy Cancer Center, Institut Gustave Roussy, 114 rue Edouard Vaillant, 94800 Villejuif, France

2 LabEx LERMIT, Université Paris Saclay, 92296 Châtenay-Malabry, France

3 Institut Gustave Roussy, Inserm, Biomarqueurs prédictifs et nouvelles stratégies thérapeutiques en oncologie, Université Paris-Saclay, 94800 Villejuif, France

4 Cell Division Laboratory, Danish Cancer Society Research Center, 2100 Copenhagen, Denmark the asters beyond a minimal distance for proper cleavage by the cytokinesis machinery at late stages of cell division [3]. Defects in spindle size and architecture can lead to mitotic defects and promote aneuploidy, which is a hallmark of cancer. Metaphase spindle length is controlled by intrinsic and extrinsic factors [3]. Poleward microtubule flux, which consists of translocation of tubulin heterodimers towards the poles, coupled to a balance between polymerization of microtubule plus ends at kinetochores and depolymerization of minus ends at the poles $[4,5]$, is a major mechanism to maintain the spindle at a constant

5 Centre de Recherche, Laboratoire de Spectrométrie de Masse Protéomique, Institut Curie, PSL Research University, 75248 Paris Cedex 05, France

6 Wellcome Trust Centre for Cell Biology, School of Biological Sciences, University of Edinburgh, Edinburgh, Scotland, UK

7 Institut de Génétique et Développement de Rennes (IGDR), Unité CNRS, UMR 6290, Université de Rennes, 35043 Rennes, France

8 Department of Cellular and Molecular Medicine, Faculty of Health Sciences, University of Copenhagen, 2100 Copenhagen, Denmark

\begin{tabular}{|l|l|l|l|l|}
\hline Journal : Large 18 & Article No : 3614 & Pages : 15 & MS Code : 3614 & Dispatch : 10-8-2020 \\
\hline
\end{tabular}


length in metaphase $[2,6]$. Microtubule-associated proteins and depolymerizing kinesins, that regulate microtubule dynamics at both ends, thus appear as important regulators of poleward flux and spindle length [3, 7-9].

The Kif2A kinesin is a key regulator of poleward microtubule flux and spindle size in drosophila, xenopus and human cells [6, 10-13]. Kif2A belongs to the kinesin-13 family of depolymerizing kinesins also including Kif2B and MCAK/Kif2C [14]. During mitosis, Kif2A localizes to spindle poles where it depolymerizes microtubule minus ends $[10,15,16]$, thereby controlling the rate of poleward microtubule flux. Transport of Kif2A to spindle poles is ensured by minus-end-directed motor protein dynein/dynactin [10] and is increased by interaction with microtubule-associated Dda3 protein, whose expression and phosphorylation are markedly elevated in mitosis [17, 18]. Depletion of either Dda3 or Kif2A increases mitotic spindle size [10, 17]. The microtubule depolymerizing activity of Kif2A and its recruitment to the poles are inhibited by Aurora kinase A (Aurora A) [19], a major mitotic kinase that localizes on spindle poles. Aurora A fulfills numerous functions $[20,21]$ including the regulation of spindle size $[13,22]$. However, the mechanisms by which this kinase controls metaphase spindle length are poorly defined. Whether the maintenance of correct spindle size in metaphase depends on Aurora A-mediated regulation of Kif2A recruitment to the poles remains to be investigated.

ATIP3 is a microtubule-associated protein that decorates the centrosome and microtubule cytoskeleton in interphase, and the mitotic spindle and spindle poles during mitosis [23]. Previous studies have shown that ATIP3 is a potent microtubule stabilizer [24] that reduces microtubule dynamics by interacting with End-Binding protein EB1 in interphase [25, 26]. ATIP3 is the product of candidate tumor suppressor gene MTUS1 whose expression is markedly down-regulated in aggressive breast tumors [23, 24]. We have recently shown that ATIP3 depletion is associated with increased aneuploidy and mitotic defects [27].

In this study, we show that ATIP3 depletion leads to shortening of the metaphase spindle. Mass spectrometry analysis identified Kif2A as an intracellular partner of ATIP3. We provide evidence that ATIP3 forms a complex with Kif2A and Dda3 and limits their recruitment to the poles, thereby contributing to maintaining constant spindle size in metaphase. The interaction between ATIP3, Dda3 and Kif2A is increased in mitosis and requires phosphorylation by Aurora kinase A. Conversely, the localization of Aurora A at spindle poles is regulated by ATIP3. Overall, we show that reciprocal regulation of ATIP3 and Aurora kinase A during mitosis ensures the timely assembly of a molecular ATIP3-Dda3-Kif2A complex that controls metaphase spindle length.

\section{Materials and methods}

\section{Cell lines and synchronization}

Human cancer cell lines MCF-7, MCF-7 cells stably expressing moderate levels of GFP-ATIP3 (HC6 clone), HCC1143 and HeLa were described previously [23, 24]. MCF-7 cells express undetectable levels of ATIP3 whereas HCC1143 and HeLa cells express endogenous ATIP3 [23, 24]. MCF-7 cells were cultured in DMEM-F12 supplemented with $5 \%$ bovine calf serum. HCC1143 cells were cultured in RMPI supplemented with $10 \%$ bovine calf serum and $1 \%$ sodium pyruvate. HeLa cells were cultured in DMEM supplemented with $10 \%$ boyine calf serum, $100 \mathrm{U} / \mathrm{ml}$ penicillin, and $100 \mathrm{U} / \mathrm{ml}$ streptomycin (all from Gibco) at $37{ }^{\circ} \mathrm{C}$ and $5 \% \mathrm{CO}_{2}$. Cells were routinely authenticated by morphologic observation and tested for absence of mycoplasma contamination using Venor ${ }^{\circledR} \mathrm{GeM}$ Advance Kit (MB Minerya biolabs ${ }^{\circledR}$ ).

For synchronization in metaphase, MCF-7 and $\mathrm{HCC} 1143$ cells were treated with $50 \mathrm{ng} / \mathrm{ml}$ nocodazole for $16 \mathrm{~h}$ and released for $45 \mathrm{~min}$.

\section{Plasmid constructs, siRNAs and transfections}

Plasmids encoding GFP-ATIP3, GFP-D1, GFP-D2, GFPD2, GFP-D2N, GFP-D2C, GFP-D3, GFP-ATIP3delCN and GFP-D2delCN were described elsewhere [24, 25]. Deletion mutants GFP-delCN2 and GFP-D2delCN2 (lacking amino acid positions 635-816 of ATIP3, accession number NP_001001924) were obtained by PCR amplification of the ATIP3 and D2 sequence, respectively, using the following oligonucleotides: 5'-GGG TCC GTT TCT GCG TTG TTT TCT GGT AAT GCC GCT GTC-3' and 5'-GAC AGC GGC ATT ACC AGA AAA CAA CGC AGA AAC GGA CCC-3' and QuikChange ${ }^{\circledR}$ II XL Site-Directed and Mutagenesis Kit (Agilent). Plasmids encoding GFP-AurA and GFP-AurA-KD were described elsewhere [28-30]. Plasmid encoding Kif2A-GFP was provided by Jamel Chelly (Institut de Génétique et de Biologie Moléculaire et Cellulaire, Strasbourg, France). All cDNA constructs were transfected $(2-4 \mu \mathrm{g})$ for $24 \mathrm{~h}$ using X-treme Gene 9 (Roche) or Lipofectamine 2000 (Invitrogen) and were expressed at levels similar to those of endogenous proteins.

Specific and control scrambled siRNAs were from Dharmacon (ThermoFisher Scientific). The following sequences were used: ATIP3\#1, 5'-UGGCAGAGGUUU AAG GUUA-3'; ATIP3\#2, 5'-GCAAAUAGCUGCUCC AAAA-3'; Kif2A, 5'-GGCAAAGAGAUUG ACCUGG-3'; Aurora A, 5'-AUGCCCUGUCUUACUGUCA-3'; Dda3, 5'-AAGCAAGACU UCAGUAGCAUU-3'. For rescue 
experiments of ATIP3 knock-down, HeLa cells were transfected with ATIP3\#1 siRNA that targets the $5^{\prime}$ untranslated sequence of ATIP3 and allows ectopic expression of the ATIP3 coding sequence [25].

All siRNAs $(50 \mathrm{nM})$ were transfected for $48-72 \mathrm{~h}$ using Lipofectamine 2000 (Invitrogen) and silencing efficiency was evaluated by immunoblotting. Antibodies used for immunoblotting were rabbit anti-ATIP3 (anti-MTUS1; ARP44419-P050; Aviva Systems, 1/1000e), rabbit antiKif2A (ab37005; Abcam, 1/5000e), mouse anti-Aurora A (ab13824; Abcam, 1/1000e), rabbit anti-Dda3 (GTX128047; GeneTex, 1/1000e), rabbit anti-GFP (6556; Abcam, 1/1000e), mouse anti-GFP (ab1218; Abcam, 1/1000e), mouse anti-alpha-tubulin (T9026; Abcam, 1/5000e), antiphospho-histone H3 (06-570; Millipore, 1/1000e), mouse anti-gamma-actin (ab123034; Abcam, 1/5000e), mouse antivinculin (V9264; Sigma, 1/5000e).

\section{Immunoprecipitations}

HeLa or MCF-7 cells were transfected as described above and lysed in lysis buffer containing $50 \mathrm{mM}$ Tris- $\mathrm{HCl} \mathrm{pH}$ 7.4, $150 \mathrm{mM} \mathrm{NaCl}, 1 \mathrm{mM}$ EDTA, $2.5 \mathrm{mM} \mathrm{MgCl} 2,0.2 \mathrm{M}$ PMSF, $1 \mathrm{mM}$ Aprotinin, $5 \mathrm{mg} / \mathrm{ml}$ Leupeptin, $2 \mathrm{mg} / \mathrm{ml}$ Pepstatin, $0.2 \mathrm{M}$ orthovanadate, $1 \mathrm{M}$ sodium fluoride, and $10 \mu \mathrm{M}$ okadaic acid. Lysates were centrifuged, incubated with uncoupled magnetic agarose beads at $4{ }^{\circ} \mathrm{C}$ for $15 \mathrm{~min}$, and then incubated with anti-GFP VHH coupled to magnetic agarose beads (GFP-trap_MA; Chromotek) at $4{ }^{\circ} \mathrm{C}$ overnight. Antibody beads were recovered with a bar magnet, washed once with the lysis buffer in the presence of antiproteases and then twice with the lysis buffer, and analyzed by Western blotting with appropriate antibodies as described above. Results shown are representative of 3-5 independent experiments.

\section{Proteomics and mass spectrometry}

MCF-7 cells were transiently transfected with $2 \mu \mathrm{g}$ of GFPATIP3 or GFP for $24 \mathrm{~h}$ then lysed as described above. For immuno-isolated samples, $1 \mathrm{mg}$ extract was incubated with $25 \mu \mathrm{l}$ magnetic beads (GFP-trap_MA; Chromotek) for $2 \mathrm{~h}$ at $4{ }^{\circ} \mathrm{C}$.

Proteins on magnetic beads were washed twice with $100 \mu \mathrm{l}$ of $25 \mathrm{mM} \mathrm{NH}_{4} \mathrm{HCO}_{3}$ and on-beads digestion were performed with $0.2 \mu \mathrm{g}$ of trypsin/LysC (Promega) for $1 \mathrm{~h}$ in $100 \mu \mathrm{l}$ of $25 \mathrm{mM} \mathrm{NH}_{4} \mathrm{HCO}_{3}$. Sample were then loaded onto a homemade $\mathrm{C} 18$ StageTips for desalting (principle by stacking one $3 \mathrm{M}$ Empore SPE Extraction Disk Octadecyl (C18) and beads from SepPak C18 CartridgeWaters into a $200 \mu 1$ micropipette tip). Peptides were eluted using 40/60 $\mathrm{MeCN} / \mathrm{H}_{2} \mathrm{O}+0.1 \%$ formic acid and vacuum concentrated to dryness.
Online chromatography was performed with an RSLCnano system (Ultimate 3000, Thermo Scientific) coupled online to an Orbitrap Fusion Tribrid mass spectrometer (Thermo Scientific). Peptides were trapped on a C18 column $(75 \mu \mathrm{m}$ inner diameter $\times 2 \mathrm{~cm}$; nanoViper Acclaim PepMapTM 100, Thermo Scientific) with buffer A (2/98 $\mathrm{MeCN} / \mathrm{H}_{2} \mathrm{O}$ in $0.1 \%$ formic acid) at a flow rate of $4.0 \mu \mathrm{l} / \mathrm{min}$ over $4 \mathrm{~min}$. Separation was performed on a $50 \mathrm{~cm} \times 75 \mu \mathrm{m}$ C18 column (nanoViper Acclaim PepMapTM RSLC, $2 \mu \mathrm{m}, 100 \AA$, Thermo Scientific) regulated to a temperature of $55{ }^{\circ} \mathrm{C}$ with a linear gradient of $2-30 \%$ buffer B $(100 \%$ $\mathrm{MeCN}$ in $0.1 \%$ formic acid) at a flow rate of $350 \mathrm{nl} / \mathrm{min}$ over 160 min. Full-scan MS was acquired in the Orbitrap analyzer with a resolution set to 120,000 and ions from each full scan were HCD fragmented and analyzed in the linear ion trap.

For identification the data were searched against the SwissProt Homo Sapiens (February 2017, no isoforms) database using Sequest HF through proteome discoverer (version 2.1). Enzyme specificity was set to trypsin and a maximum of two missed cleavage site were allowed. Oxidized methionine, $\mathrm{N}$-terminal acetylation, and carbamidomethyl cysteine were set as variable modifications. Maximum allowed mass deviation was set to $10 \mathrm{ppm}$ for monoisotopic precursor ions and 0.6 Da for MS/MS peaks.

The resulting files were further processed using myProMS [31] v3.6 (work in progress). FDR calculation used Percolator and was set to $1 \%$ at the peptide level for the whole study. The label free quantification was performed by peptide Extracted Ion Chromatograms (XICs) computed with MassChroQ version 2.2.2 [32]. For protein quantification, XICs from proteotypic peptides shared between compared conditions (TopN matching) with no missed cleavages were used. Median and scale normalization was applied on the total signal to correct the XICs for each biological replicate. To estimate the significance of the change in protein abundance, a linear model (adjusted on peptides and biological replicates) was performed and $p$-values were adjusted with a Benjamini-Hochberg FDR procedure with a control threshold set to 0.05 . Fold change-based GO enrichment analysis was performed as described [33].

The mass spectrometry proteomics data have been deposited to the ProteomeXchange Consortium via the PRIDE [34] partner repository with the dataset identifier PXD009182 (username: reviewer03478@ebi.ac.uk, password: UFFpYuYZ).

\section{Immunofluorescence}

Cells were fixed on cover glasses with ice-cold methanol for 5 min and washed with PBS. Coverslips were subsequently incubated at room temperature for $1 \mathrm{~h}$ in primary antibodies and for $1 \mathrm{~h}$ in Alexa Fluor 488-, Alexa Fluor 549- and Alexa Fluor 647-coupled secondary antibodies diluted in PBS/

\begin{tabular}{|l|l|l|l|l|}
\hline Journal : Large 18 & Article No : 3614 & Pages : 15 & MS Code : 3614 & Dispatch : 10-8-2020 \\
\hline
\end{tabular}



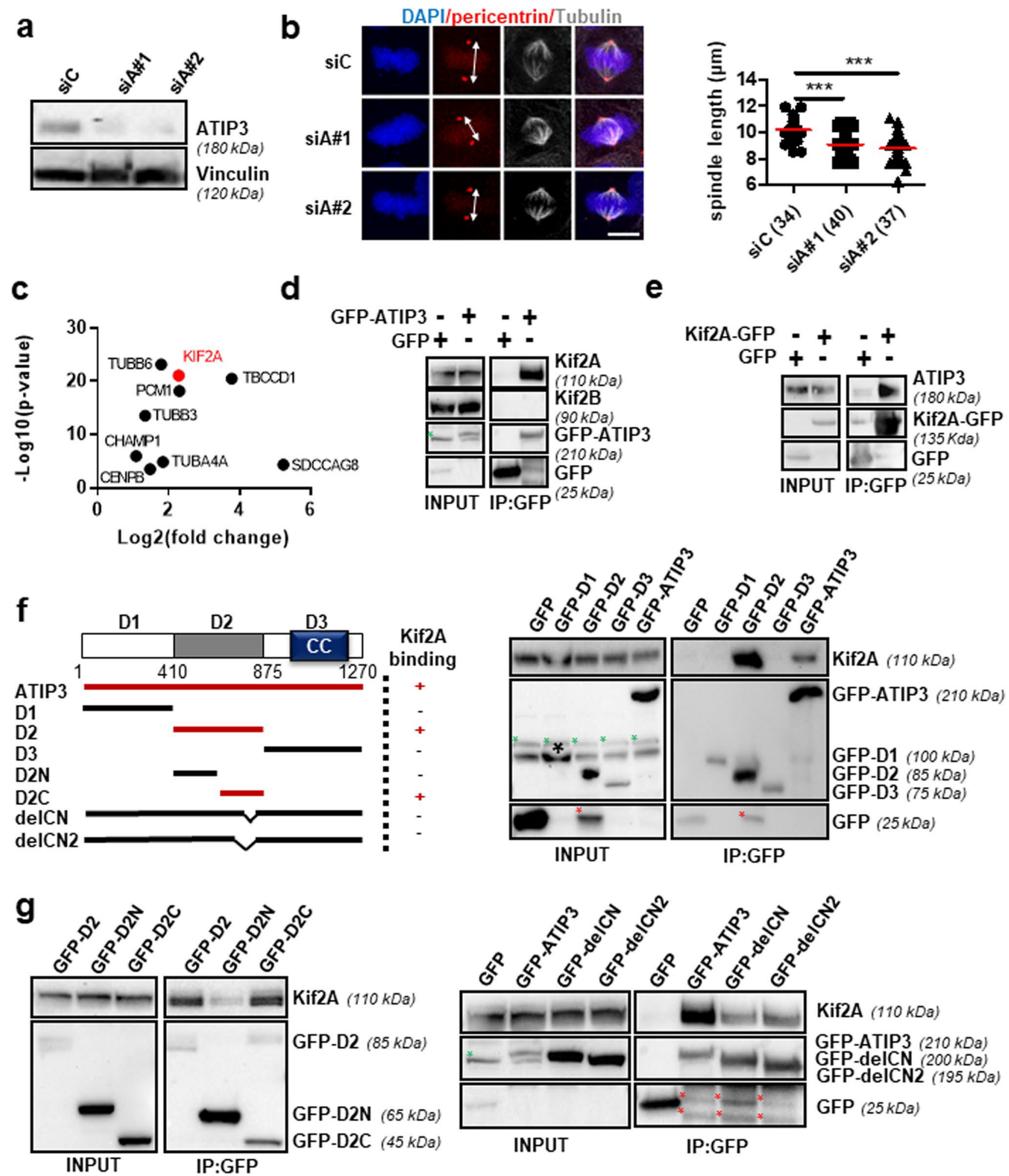

Triton $0.1 \%$, BSA $0.2 \%$. The following primary antibodies were used: rabbit anti-Kif2A (ab37005; Abcam, 1/250e), rabbit anti-pericentrin (ab4448; Abcam, 1/500e), rabbit antiDda3 (GTX128047; GeneTex, 1/500e), mouse anti-Aurora A (ab13824; Abcam, 1/200e), rabbit Phospho-Aurora A
(Thr288) (3079S; Cell Signaling, 1/200e), mouse anti-GFP (ab1218; Abcam, 1/250e), rat anti-alpha-tubulin (ab6160; Abcam, 1/500e), mouse anti-gamma-tubulin (T5326; Sigma, 1/500e) and mouse anti-alpha- tubulin (T9026; Sigma, 1/100e). Coverslips were fixed with Glycergel Mounting

\begin{tabular}{|l|l|l|l|l|}
\hline Journal : Large 18 & Article No : 3614 & Pages : 15 & MS Code : 3614 & Dispatch : 10-8-2020 \\
\hline
\end{tabular}


4Fig. 1 ATIP3 interacts with Kif2A. a Immunoblotting with antiATIP3 (MTUS1) antibodies shows silencing efficiency of two different ATIP3-specific siRNAs (siA\#1 and siA\#2) transfected in HeLa cells. Blots were reprobed with anti-vinculin antibodies for internal control. b Immunofluorescence imaging of HeLa cells transfected with control (siC), and two different ATIP3-specific siRNAs (siA\#1 and siA\#2) as indicated. Cells were stained with anti-pericentrin (red), anti-alpha-tubulin (gray) antibodies and DAPI (blue). Scale bar, $10 \mu \mathrm{m}$. Pole-to pole distance (white arrow) was measured by drawing a line with ImageJ software between the two poles stained with pericentrin. Right panel shows quantification of spindle length in metaphase. Number of cells analyzed is under brackets. $* * * p<0.001$. c Volcano plot showing distribution of the 9 proteins related to the microtubule cytoskeleton and/or mitosis present in GFP-ATIP3 compared with GFP and identified by mass spectrometry. $x$ axis $=\log 2$ (fold-change), $y$ axis $=-\log 10$ ( $p$-value). Position of the ATIP3 partner Kif2A is indicated in red. d MCF-7 cells were transfected with GFP-ATIP3 or GFP and immunoprecipitation was performed using anti-GFP antibodies. Western blots were probed with anti-Kif2A, anti-Kif2B, anti-ATIP3 and anti-GFP antibodies to reveal Kif2A, Kif2B, GFP-ATIP3 and GFP, respectively. A green asterisk in the INPUT panel indicates endogenous ATIP3. e HeLa cells were transfected with Kif2A-GFP or GFP and immunoprecipitation was performed using anti-GFP antibodies. Western blots were probed with anti-ATIP3 (MTUS1) and anti-GFP antibodies to reveal ATIP3, Kif2A-GFP and GFP. f Schematic representation of the ATIP3 protein sequence illustrating the position of D1, D2 and D3 regions and ATIP3 deletion mutants and their ability (+) or not (-) to bind Kif2A. Kif2A-binding regions are in red. CC: coiled coil region. Amino acid numbering is from accession number NP_001001924. Right panel: immunoprecipitation assay of MCF-7 cell lysates expressing GFP, GFP-ATIP3 or GFP-fused D1, D2, D3 regions. Blots were probed with anti-Kif2A and anti-GFP antibodies. A green asterisk indicates non-specific band. A black star indicates the position of GFP-D1 which migrates at the same apparent molecular weight as endogenous Kif2A revealed in previous blotting. A red asterisk indicates cleavage product of GFP-D2. g MCF-7 cells were transfected with GFP-D2 fragments (left panel) or GFP-ATIP3 deletion mutants (right panel) and immunoprecipitation was performed using anti-GFP antibodies. Blots were probed as in d. A green asterisk shows endogenous ATIP3. Red asterisks indicate cleavage products of fusion proteins
Medium (Dako) coupled with DAPI. Images were acquired with a confocal laser scanning microscope Dmi8- SP8 with an HC PL APO CS2 40x-1.3 Oil (Leica). Linescan analyses of pole-to-pole distance and fluorescence intensities were done with Image J. At least 30 cells in 4-10 fields per condition were analyzed.

Results shown are representative of 3-5 independent experiments.

\section{Fluorescence recovery after photobleaching (FRAP)}

HeLa cells were transfected with control or ATIP3 siRNA and co-transfected with Kif2A-GFP. For the experiment, cells were seeded in $35 \mathrm{~mm}$ glass-bottomed dishes (14 mm, No. 1.5, MatTek Corporation). The siRNA transfection was done $72 \mathrm{~h}$ prior to filming using Lipofectamine 2000 (Invitrogen). Kif2A-GFP was co-transfected $48 \mathrm{~h}$ after siRNA transfection. $10 \mathrm{nM}$ SiR-DNA (Spirochrome) was added to the cell culture medium 30 min before starting the live-cell imaging. Time-lapse imaging was performed at $37^{\circ} \mathrm{C}$ using a Plan-Apochromat 63x/1.4NA with differential interference contrast oil objective mounted on an inverted Zeiss Axio Observer Z1 microscope (Marianas Imaging Workstation from Intelligent Imaging and Innovations Inc. (3i), Denver, CO, USA), equipped with a CSU-X1 spinning-disk confocal head (Yokogawa Corporation of America). Images were acquired using an iXon Ultra 888 EM-CCD camera (Andor Technology). After identification of spindle poles in metaphase cells, one of the two poles was photobleached with a $5 \mathrm{~ms}$ laser pulse using $100 \%$ power of the $488 \mathrm{~nm}$ solid state laser, and the fluorescence recovery was recorded for $90 \mathrm{~s}$ at $500 \mathrm{~ms}$ intervals. For FRAP calculations, the fluorescence intensity of the bleached area was normalized using the intensity of the whole cell, after background correction. Then a full-scale normalization was performed and the mean values were plotted and used for exponential curve fitting using GraphPad Prism 7.01 to determine the half-life and efficiency of the recovery.

\section{Poleward microtubule flux rate measurement}

Photo-conversion of alpha-tubulin was performed using $\mathbf{A Q 3}_{4}$ a stable U2OS-mEOS-tubulin cell line [35] cultivated on 285 $35 \mathrm{~mm}$ glass-bottomed dishes (14 mm, No. 1.5, MatTek 286 Corporation). Transfection of control or ATIP3 siRNA 287 was done $48 \mathrm{~h}$ prior to filming using Lipofectamine $2000 \quad 288$ (Invitrogen). Time-lapse imaging was performed at $37{ }^{\circ} \mathrm{C} \quad 289$ using a Plan-Apochromat 63x/1.4NA oil objective with 290 differential interference contrast mounted on an inverted Zeiss Axio Observer Z1 microscope (Marianas Imaging Workstation from Intelligent Imaging and Innovations Inc. (3i), Denver, CO, USA), equipped with a CSU-X1 spinning-disk confocal head (Yokogawa Corporation of America) and a photo-activation system with $405 \mathrm{~nm}$ laser line. Images were acquired using an iXon Ultra 888 EMCCD camera (Andor Technology). Late prometaphase/ metaphase cells were identified upon green fluorescent tubulin signals and two line-shaped regions of interest were placed perpendicular to the main spindle axis on both sides of the metaphase plate to be photo-activated. Photo-conversion was performed by a 5 -ms $405-\mathrm{nm}$ laser pulse. Photo-converted red signals were then followed over time together with green fluorescence signal using 561and 488-nm lasers and images were acquired every $5 \mathrm{~s}$ for $3 \mathrm{~min}$. Microtubule flux was quantified by tracking photoconverted mEos-tubulin over time using sum-projected kymographs generated by a MATLAB-based algorithm [36].
291

292

293

294

295

296

297

298

299

300

301

302

303

304

305

306

307

308

309

310

\begin{tabular}{|l|l|l|l|l|}
\hline Journal : Large 18 & Article No : 3614 & Pages : 15 & MS Code : 3614 & Dispatch : 10-8-2020 \\
\hline
\end{tabular}




\section{Statistical analysis}

Statistical analyses were done using GraphPad Prism 6.0 software. Data in bar graphs (mean $\pm \mathrm{SD})$ and dot plots were analyzed using two-tail unpaired $t$ test and ANOVA test. $p<0.05$ was considered statistically significant.

\section{Results}

\section{ATIP3 depletion reduces metaphase spindle length}

To evaluate the consequences of ATIP3 depletion in mitosis, HeLa cells were transfected with two independent ATIP3 siRNAs (siA\#1 and siA\#2) (Fig. 1a) and mitotic cells were analyzed by immunofluorescence. By measuring pole-to-pole distance in metaphase cells using pericentrin as a marker, we observed that ATIP3 depletion with each siRNA reduces spindle length (Fig. 1b). Similar results were obtained using both ATIP3 siRNAs in HCC1143 breast cancer cells (Fig. S1A). Conversely, moderate expression of GFP-ATIP3 in ATIP3-negative MCF-7 cells led to an increase in spindle size compared to control cells expressing GFP (Fig. S1B).

Other mitotic defects such as multipolar spindles (Fig. S1C) and spindle mispositioning (Fig. S1D) were observed in ATIP3-depleted cells but spindle orientation, symmetry and cell size remained unchanged (Fig. S1E).

\section{ATIP3 interacts with Kif2A}

In a first step to elucidate the molecular mechanisms by which ATIP3 controls spindle size, we undertook a proteomic approach to identify novel intracellular ATIP3 binding partners. Anti-GFP antibodies were used to immunoprecipitate molecular complexes from MCF-7 breast cancer cells expressing either GFP or GFP-ATIP3 fusion protein. Mass spectrometry analysis allowed us to identify 145 proteins that selectively interact with ATIP3 (Table S1), nine of which were related to the microtubule cytoskeleton and/or mitosis (Fig. 1c, Table 1). These include 3 tubulin chains (beta6, alpha4 and beta3), 2 centromere/kinetochore associated proteins (CENPB, CHAMP1) and 4 centrosomal/spindle pole proteins (SDCCAG8, TBBCD1, PCM1 and Kif2A). The Kif2A kinesin was among the best candidate ATIP3 partners in terms of fold change (4.86) and $p$ value $(8.14 \mathrm{e}-22)$. This depolymerizing kinesin is also a well-known regulator of mitotic spindle length [10] and was therefore selected for further studies.

The interaction between ATIP3 and Kif2A was confirmed by co-immunoprecipitation analyses. GFP-ATIP3 fusion protein expressed in MCF-7 cells was immunoprecipitated using anti-GFP antibodies and found to bind endogenous Kif2A (Fig. 1d). Kinesin Kif2B was not retained in GFP-ATIP3 immunocomplexes, indicating specificity. Conversely, endogenous ATIP3 was found to interact with Kif2A-GFP in HeLa cells (Fig. 1e), confirming the existence of ATIP3-Kif2A molecular complexes. As shown in Fig. S1F, ATIP3 accumulates along the entire length of the spindle, including the spindle poles where Kif2A is localized.

To better characterize the interaction, we analyzed three truncated constructs designated D1, D2 and D3 (Fig. 1f) encompassing the $\mathrm{N}$-terminal acidic region, the central MT-binding domain and the C-terminal coiled-coil region of ATIP3, respectively [24]. After transient transfection of

Table 1 ATIP3-interacting partners related to microtubule cytoskeleton and/or mitosis

\begin{tabular}{lclll}
\hline Gene and synonyms & \multicolumn{2}{l}{ ATIP3/GFP } & \multicolumn{2}{l}{ Description } \\
\cline { 2 - 4 } & Ratio & Log2 & $p$-value & \\
\hline MTUS1 & 400.563902 & 8.6458886 & $4.2716 \mathrm{E}-51$ & Microtubule-associated tumor suppressor 1 \\
SDCCAG8 & 37.6347222 & 5.23399242 & $4.7636 \mathrm{E}-05$ & Serologically defined colon cancer antigen 8 \\
TBCCD1 & 13.6959029 & 3.77567248 & $3.2558 \mathrm{E}-21$ & TBCC domain-containing protein 1 \\
PCM1 & 4.92790341 & 2.30097398 & $6.9899 \mathrm{E}-19$ & Pericentriolar material 1 protein \\
KIF2A & 4.86820741 & 2.28339063 & $8.1438 \mathrm{E}-22$ & Kinesin-like protein KIF2A \\
CENPB & 3.58061441 & 1.84020717 & $1.35 \mathrm{E}-05$ & Major centromere autoantigen B \\
TUBB6 & 3.45212501 & 1.78748471 & $6.9563 \mathrm{E}-24$ & Tubulin beta-6 chain \\
TUBA4A & 2.78607967 & 1.47823652 & 0.00032993 & Tubulin alpha-4A chain \\
TUBB3 & 2.53211069 & 1.34034047 & $2.8227 \mathrm{E}-14$ & Tubulin beta-3 chain \\
CHAMP1 & 2.12271217 & 1.08590876 & $1.0626 \mathrm{E}-06$ & Chromosome alignment-maintaining phosphoprotein 1 \\
\hline
\end{tabular}

MCF-7 cells were transfected with GFP or GFP-ATIP3 and cell lysates were precipitated using GFP-trap magnetic agarose beads. Proteins retained in immunocomplexes were analyzed by quantitative label-free mass spectrometry analysis performed on four replicates. Shown are the fold changes of 9 proteins related to microtubules and/or mitosis that were selected among 145 proteins quantified by label-free quantitative analysis of GFP-ATIP3 versus GFP according to absolute fold change $\geq 2$, adjusted $p$-value of ratio significance $\leq 0.05$ and more than 3 peptides. Proteins are listed by ratio order

\begin{tabular}{|l|l|l|l|l|}
\hline Journal : Large 18 & Article No : 3614 & Pages : 15 & MS Code : 3614 & Dispatch : 10-8-2020 \\
\hline
\end{tabular}


GFP-tagged constructs followed by co-immunoprecipitation, results showed that the central region of 410 amino-acids (D2 region) interacts with Kif2A (Fig. 1f, right panel). Truncated mutants of the $\mathrm{D} 2$ region (D2N, D2C) were then analyzed. The $\mathrm{D} 2 \mathrm{C}$ sequence encompassing amino acid residues 705-874 of ATIP3 was sufficient to bind Kif2A (Fig. 1g, left panel). Deletion of amino acids 705-816 (GFP-delCN) and 635-816 (GFP-delCN2) in the ATIP3 sequence led to a marked decrease in Kif2A interaction (Fig. 1g, right panel). Deleting the same sequences from the D2 polypeptide also impaired the interaction (Fig. S2A), therefore indicating that a minimal sequence of 112 amino acids ( $\mathrm{CN}$ sequence) is required for ATIP3 interaction with Kif2A.

\section{ATIP3-Kif2A complex controls metaphase spindle length}

To investigate the functional impact of ATIP3-Kif2A complex in the control of mitotic spindle length, we investigated whether GFP-ATIP3 construct and deletion mutants were able to rescue the ATIP3 depletion phenotype. RNAiresistant GFP-ATIP3 expressed at moderate levels in ATIP3depleted cells indeed restored a spindle size similar to that observed in control cells (Fig. 2a). In contrast, deletion mutants GFP-DelCN and GFP-DelCN2 - that are unable to interact with Kif2A—failed to rescue the phenotype (Fig. 2a, S2B). Furthermore, expressing the Kif2A-interacting region D2 was sufficient to restore a correct spindle length in ATIP3-depleted cells whereas Kif2A-binding defective mutants D2delCN and D2delCN2 were not (Fig. S2C). Together these data strongly suggest that ATIP3 may control metaphase spindle size by interacting with Kif2A.

\section{ATIP3 depletion increases Kif2A targeting to the spindle poles}

Immunofluorescence studies revealed that ATIP3 depletion markedly increases Kif2A fluorescence intensity at spindle poles (Fig. 2b) with no effect on the cytosolic pool of Kif2A (Fig. S2D). In contrast, ATIP3 depletion did not significantly modify fluorescence intensity of pericentrin, a major spindle pole protein (Fig. S2E). Expression of RNAi-resistant GFPATIP3 in ATIP3-depleted cells rescued the phenotype and decreased Kif2A intensity at the poles (Fig. 2c). Of note, ATIP3 silencing had no effect on total Kif2A protein levels detected by western blotting (Fig. 2b, left panel), suggesting that increased fluorescence intensity of Kif2A may reflect increased recruitment to the poles rather than increased expression of the protein.

We then evaluated whether increased Kif2A accumulation at spindle poles may contribute to the phenotype of ATIP3 deficiency. Silencing of Kif2A in ATIP3-depleted cells (Fig. S2F) indeed rescued the phenotype and restored a correct spindle size (Fig. 2d), indicating that increased Kif2A recruitment to the poles is responsible for spindle length shortening in ATIP3-depleted cells.

It has been previously shown that Kif2A regulates metaphase spindle length by controlling poleward microtubule flux via its microtubule depolymerizing activity at the spindle poles $[6,10,11]$. We reasoned that ATIP3 deficiency may disrupt the balance of microtubule dynamics in the spindle by allowing increased Kif2A depolymerizing activity at the poles, resulting in decreased poleward flux and shorter spindle length. To address that question, microtubule flux rates were analyzed following ATIP3 silencing in metaphase U2OS cells stably expressing alpha-tubulin fused to the photo-convertible fluorescent protein mEos2 [35]. Results indicate that poleward microtubule flux is significantly decreased in ATIP3-depleted cells $(0.53 \pm 0.13 \mu \mathrm{m} /$ min) compared to control cells $(0.67 \pm 0.08 \mu \mathrm{m} / \mathrm{min}$, $p<0.0001$ ) (Fig. 2e), supporting the notion that ATIP3 may control spindle size by regulating the recruitment-and thus, the depolymerizing activity-of Kif2A to the poles. Collectively, these data indicate that ATIP3 controls metaphase spindle length by interacting with the Kif2A kinesin and reducing its recruitment to the poles.

\section{ATIP3-Kif2A interaction involves Dda3}

How does ATIP3 silencing increase Kif2A targeting at the spindle poles? We hypothesized that ATIP3 may sequester Kif2A in the cytosol and slower the exchange rate between spindle pole-associated Kif2A and a diffuse cytosolic pool of the protein. We thus explored the dynamic behavior of Kif2A turnover on spindle poles in the presence or absence of ATIP3. Fluorescence recovery after photobleaching (FRAP) experiments were performed on metaphase HeLa cells expressing endogenous ATIP3 and transfected with Kif2A-GFP. Bleaching was done on one of the two spindle poles. We found that in control cells, Kif2A-GFP association with spindle poles is dynamic. Ninety percent of the signal was recovered after photobleaching, with a half-recovery time of $11.6 \mathrm{~s}$ (Fig. 3a) which is faster than that (30.7 s) reported for NuMA, another major spindle pole-associated protein [37]. In ATIP3-depleted cells, Kif2A-GFP fluorescence recovery parameters remained unchanged (half-recovery time of $11.62 \mathrm{~s}$ ), ruling out the hypothesis that ATIP3 depletion may modify the exchange rate between cytosolic and spindle pole-associated pools of Kif2A.

We then investigated whether ATIP3 may regulate Kif2A localization by a mechanism involving Dda3, a microtubule-associated protein known to interact with and increase Kif2A targeting to spindle poles [17]. As shown in Fig. 3b, Dda3 silencing in ATIP3-depleted cells rescued Kif2A fluorescence intensity at spindle poles, indicating that Dda3 contributes to the regulatory effects of ATIP3

\begin{tabular}{|l|l|l|l|l|}
\hline Journal : Large 18 & Article No : 3614 & Pages : 15 & MS Code : 3614 & Dispatch : 10-8-2020 \\
\hline
\end{tabular}




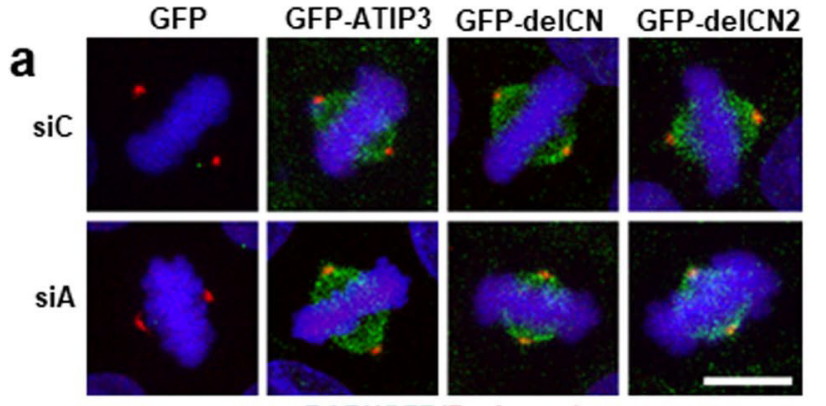

DAPI/GFP/Pericentrin

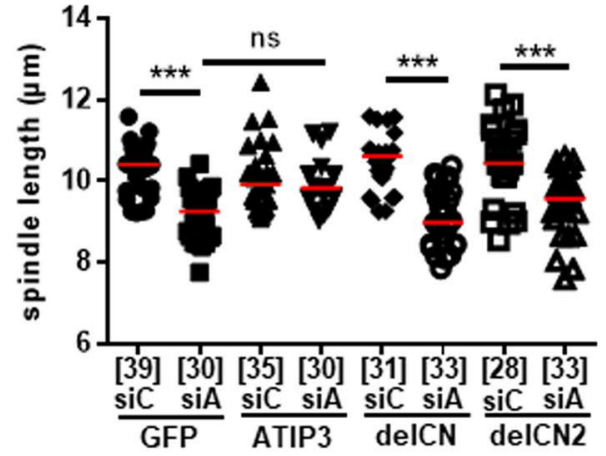

b
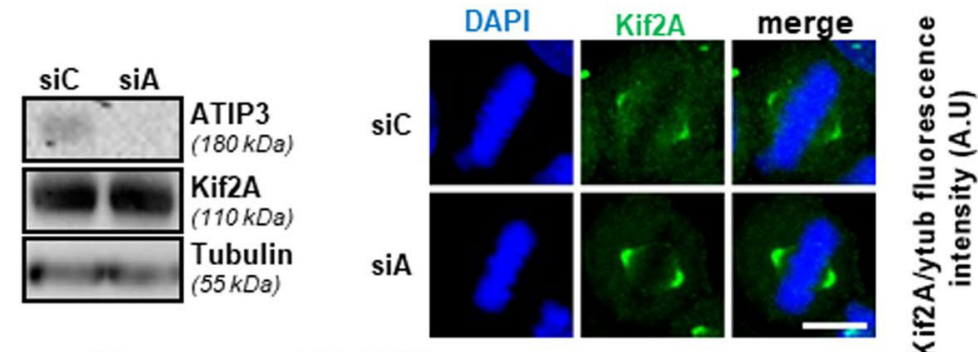

C
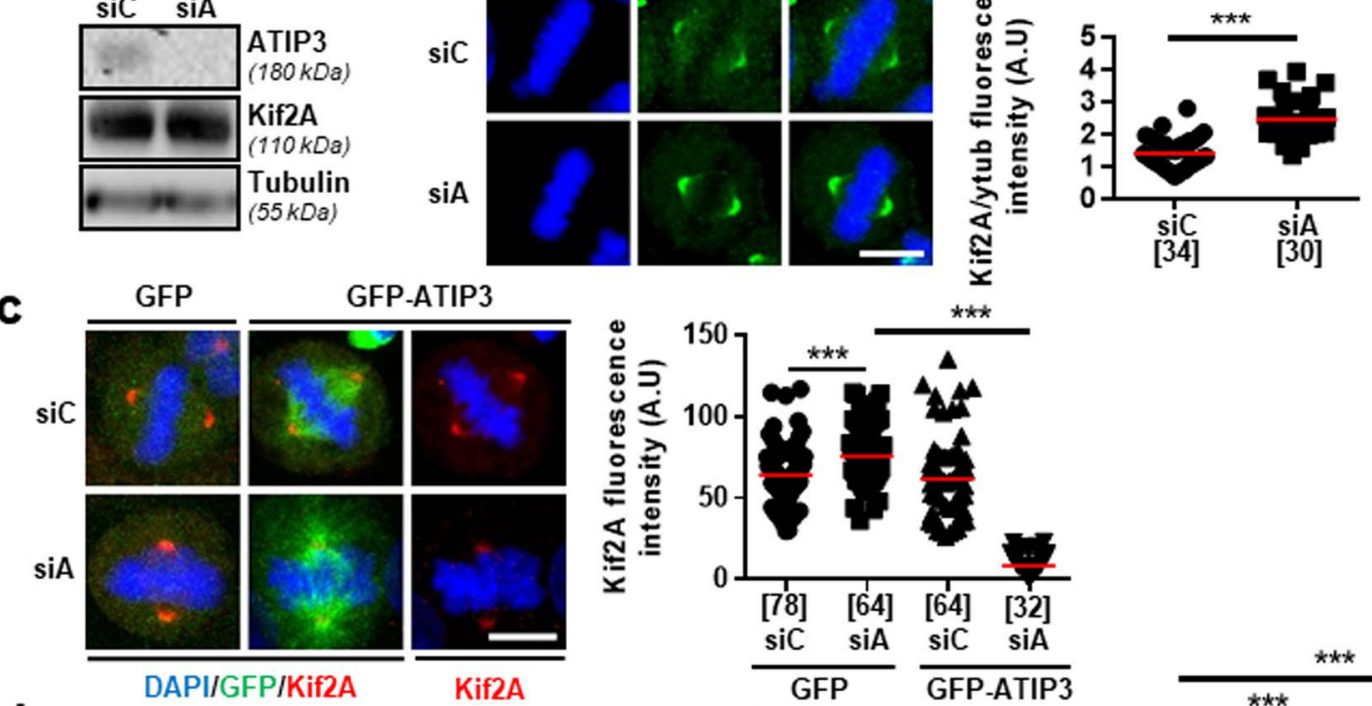

d

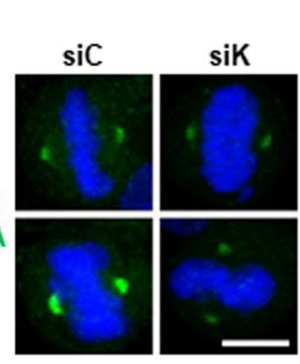

e
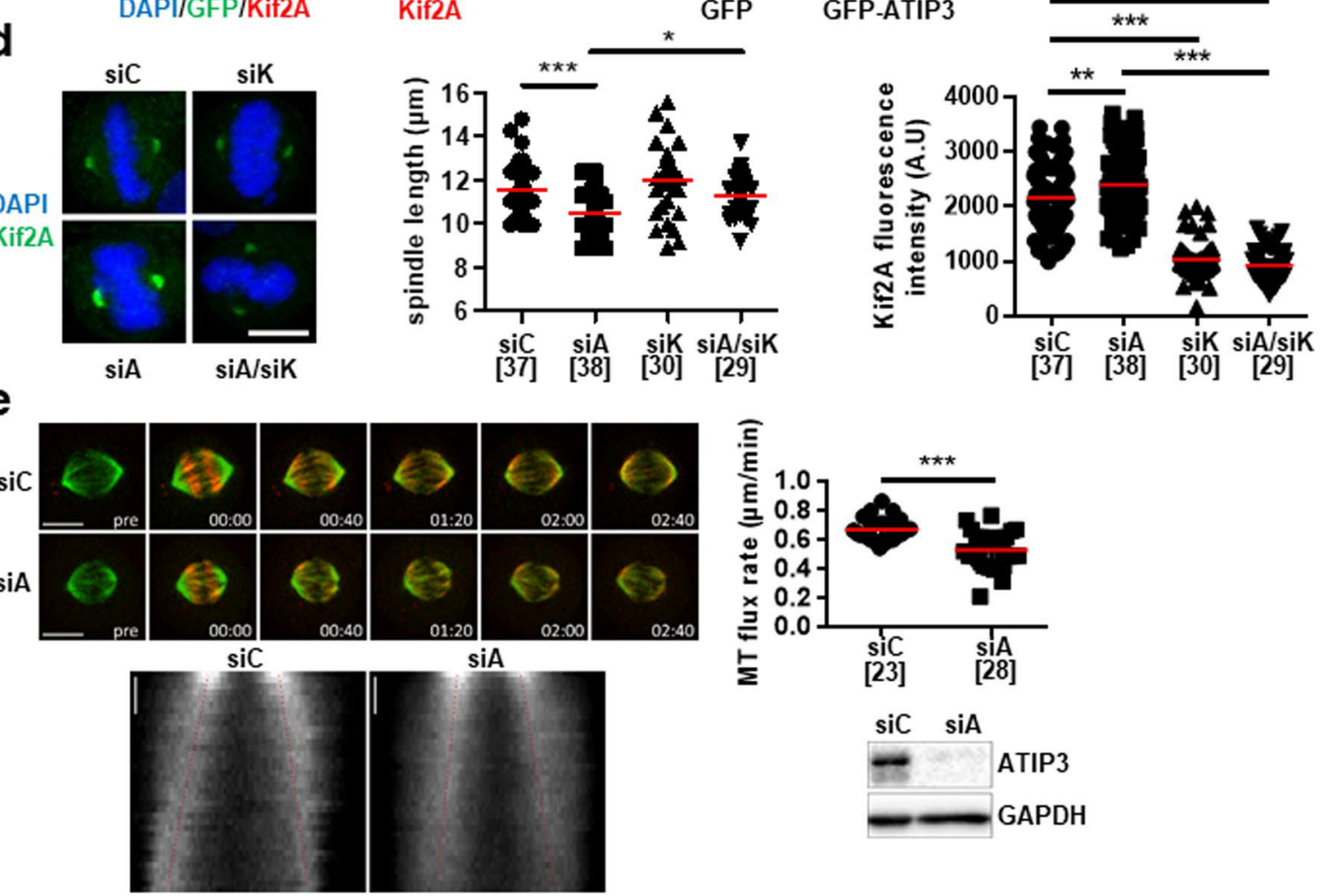
४Fig. 2 ATIP3 regulates Kif2A recruitment to spindle poles. a Immunofluorescence imaging of HeLa cells transfected for $48 \mathrm{~h}$ with control (siC) or ATIP3-specific (siA) siRNA as indicated, then for $24 \mathrm{~h}$ with GFP, GFP-ATIP3, or ATIP3 deletion mutants (GFP-delCN and GFP-delCN2) as indicated. Cells were stained with anti-GFP (green) and anti-pericentrin (red) antibodies and DAPI (blue). Scale bar, $10 \mu \mathrm{m}$. Spindle length was measured as in Fig. 1b. Right panel shows quantification of spindle length in metaphase HeLa cells. Number of cells analyzed is under brackets. $* * * p<0.001$, ns: not significant. b Left panel: Immunoblotting of HeLa cell lysates following transfection with control (siC) or ATIP3-specific (siA) siRNA as indicated. Blots were probed with anti-MTUS1 (ATIP3), anti-Kif2A and antitubulin antibodies. Middle panel: Immunofluorescence imaging of HeLa cells transfected with control (siC) or ATIP3-specific (siA) siRNA as indicated. Cells were fixed and stained with anti-Kif2A (green) antibodies and DAPI (blue). Scale bar, $10 \mu \mathrm{m}$. Kif2A fluorescence intensity was measured by drawing a line with ImageJ software between the two poles and the maximum intensity values at the poles were collected and normalized to the intensity of gamma-tubulin. Right panel: Quantification of Kif2A intensity in metaphase HeLa cells. Number of poles analyzed is under brackets. $* * * p<0.001$. c Immunofluorescence imaging of Hela cells transfected with control or ATIP3-specific siRNA for $48 \mathrm{~h}$, then were transfected for $24 \mathrm{~h}$ with GFP or GFP-ATIP3, fixed and stained with anti-Kif2A (red) and anti-GFP (green) antibodies and DAPI (blue). Scale bar, $10 \mu \mathrm{m}$. Right panel shows quantifications of Kif2A intensity at poles in metaphase HeLa cells. Number of poles analyzed is under brackets. *** $p<0.001$. d Immunofluorescence imaging of HeLa cells transfected with control (siC), ATIP3-specific (siA) and/or Kif2A-specific (siK) siRNA as indicated, and stained as in b. Scale bar, $10 \mu \mathrm{m}$. Middle panel: Spindle length (pole-to-pole distance) was measured as in Fig. 1b. Number of metaphase cells analyzed is under brackets. Right panel: Quantification of Kif2A intensity in metaphase HeLa cells as in $\mathbf{b}$. Number of poles analyzed is under brackets. $* p<0.05$, $* * p<0.01, * * * p<0.001$. e Upper left panel: Representative fluorescence images of U2OS-mEOS-tubulin cells in late prometaphase/ metaphase transfected with control (siC) and ATIP3 (siA) siRNAs. Images were captured before (pre) and after photo-conversion of mEOS-tubulin. Microtubule flux was measured by photoconversion of line-like regions from both half-spindles in mEos-tubulin cells and tracking of photoconverted mEOS-tubulin over a time interval of 3 min. Scale bar, $10 \mu \mathrm{m}$; time, min:s. Lower left panel: Representative sum-projected kymographs of photoactivated regions used for quantification of flux rates in control (siC) and ATIP3-depleted (siA) cells. Red dashed line indicates the slope of mEOS tubulin translocation over time. Scale bar, 30 s. Upper right panel: Quantification of microtubule flux in control versus ATIP3-depleted cells. Number of cells analyzed is under brackets. $* * * p<0.001$. Lower right panel: Immunoblotting-based validation of ATIP3 siRNA efficiency

on Kif2A recruitment. Accordingly, Dda3 silencing also restored a correct spindle length in ATIP3-depleted cells (Fig. 3b), indicating that Dda3 is essential to the ATIP3 phenotype. Immunofluorescence analyses revealed that, as for Kif2A, Dda3 fluorescence intensity at the poles is increased upon ATIP3 depletion (Fig. 3c). Conversely, expression of GFP-ATIP3 led a decrease of Dda3 recruitment to the poles compared with GFP (Fig. 3d). Of note, silencing of either Kif2A or Dda3 had no significant effect on ATIP3 fluorescence intensity (Fig. S3), pointing to ATIP3 as an upstream regulator of Kif2A and Dda3 targeting to the poles.
Dda3 is a known partner of Kif2A, raising the possibility that it may be part of the ATIP3-Kif2A complex. Immunoprecipitation studies revealed that Dda3 indeed interacts with ATIP3 (Fig. 3e). To evaluate whether Dda3 may compete with, or contribute to, ATIP3-Kif2A interaction, Dda3 was silenced using siRNA prior to immunoprecipitation with GFP-ATIP3. Results showed that Dda3 depletion impaired Kif2A binding to GFP-ATIP3 immunocomplexes (Fig. 3f) indicating that Dda3 is required for ATIP3-Kif2A interaction.

\section{The interaction between ATIP3, Kif2A and Dda3 is regulated in mitosis}

Dda3 levels have been shown to be elevated in mitosis [17] leading us to investigate whether ATIP3-Dda3 interaction may also be regulated in mitosis. Co-immunoprecipitation studies performed in synchronized MCF-7 cells expressing GFP-ATIP3 indeed revealed that the interaction between ATIP3 and Dda3 is increased in mitosis (Fig. 3g). Importantly, Kif2A binding to GFP-ATIP3 was also markedly increased in synchronized cells although Kif2A levels remained unchanged (Fig. 3g), which further supports the notion that Dda3 is essential to the ATIP3-Kif2A complex. Data showing Dda3 hyperphosphorylation in mitosis [38] prompted us to examine whether the interaction between ATIP3, Dda3 and Kif2A may be regulated by phosphorylation. Treatment with lambda phosphatase prior to immunoprecipitation with GFP-ATIP3 totally abrogated both Dda3and Kif2A-binding to GFP-ATIP3 (Fig. S4A), indicating that protein phosphorylation is required for ATIP3-Dda3Kif2A interaction.

\section{Aurora kinase A plays a pivotal role in the ATIP3 phenotype}

Mitosis is tightly regulated by mitotic kinases among which Aurora kinase A (Aurora A), that is localized to the poles of the spindle and whose expression is markedly increased in G2/M. Aurora A has been shown to phosphorylate both Dda3 [18, 38] and Kif2A [19] and to decrease the recruitment and microtubule-depolymerizing activity of Kif2A at the spindle poles [19]. We thus investigated whether Aurora A may be a master kinase regulating ATIP3-Dda3-Kif2A interaction.

Treatment with Aurora A kinase inhibitor MLN8054 impaired both ATIP3-Kif2A and ATIP3-Dda3 interactions (Fig. 4a), indicating that Aurora A kinase activity is required for ATIP3 interaction with Kif2a and Dda3. Aurora A depletion by siRNA also markedly decreased ATIP3-Kif2A interaction (Fig. S4B). Co-immunoprecipitation studies revealed that Aurora A binds to GFP-ATIP3 (Fig. S4C). Of note, ATIP3/Aurora A complexes were not always clearly

\begin{tabular}{|l|l|l|l|l|}
\hline Journal : Large 18 & Article No : 3614 & Pages : 15 & MS Code : 3614 & Dispatch : 10-8-2020 \\
\hline
\end{tabular}



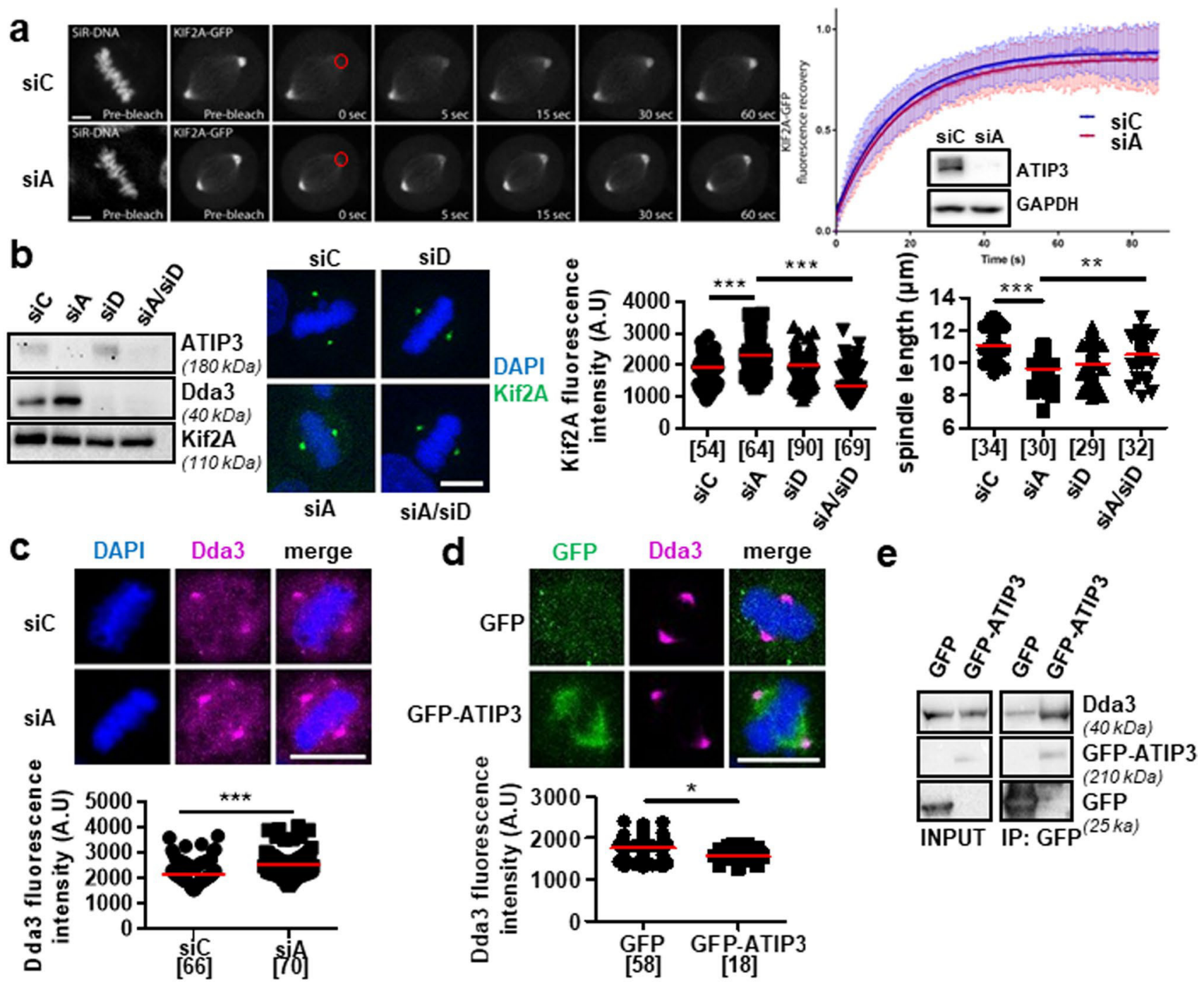

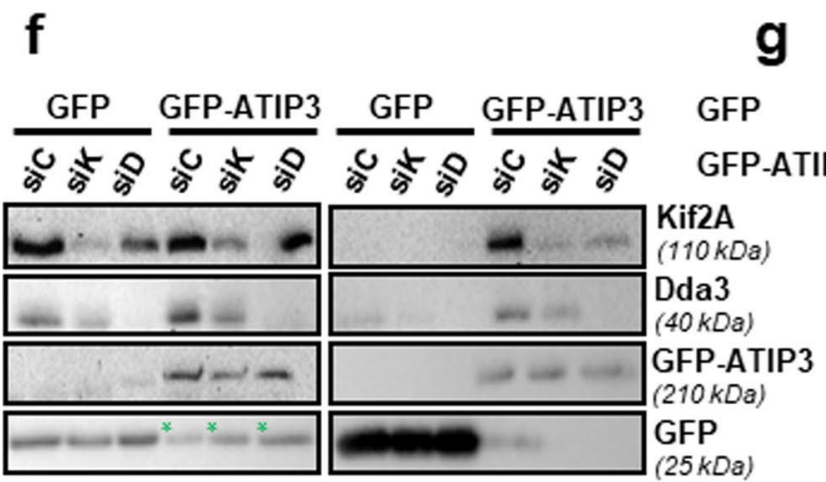

INPUT

IP : GFP

(25 kDa)

detectable, suggesting that this interaction may be weak and/

In addition to disrupting the interaction of ATIP3 with Kif2A and Dda3, Aurora A inactivation by silencing or pharmacological inhibition mimics the phenotype of ATIP3 depletion regarding Kif2A recruitment to the pole

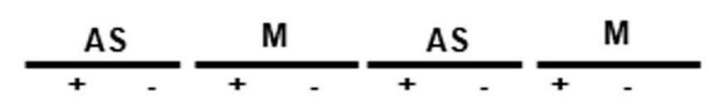

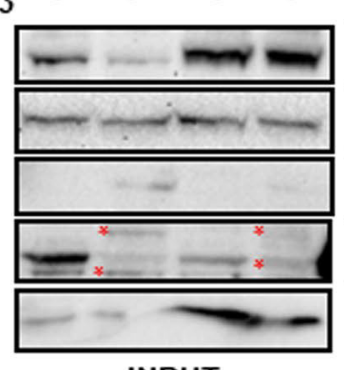

INPUT

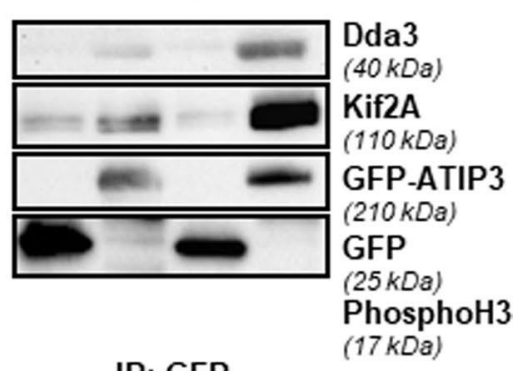

IP: GFP or transient. and regulation of spindle size (Fig. S4D, S4E). This led us to investigate whether Aurora A itself may be regulated by ATIP3. Immunofluorescence analyses revealed that ATIP3 silencing decreases Aurora A fluorescence intensity at spindle microtubules and poles (Figs. 4b, S4F), with no significant change in total protein levels detected by western

\begin{tabular}{|l|l|l|l|l|}
\hline Journal : Large 18 & Article No : 3614 & Pages : 15 & MS Code : $\mathbf{3 6 1 4}$ & Dispatch : 10-8-2020 \\
\hline
\end{tabular}


4Fig. 3 ATIP3-Kif2A interaction involves Dda3. a Left panel: Representative spinning-disk confocal live-cell images of HeLa cells in metaphase, expressing Kif2A-GFP and co-stained with SiR-DNA. Red circle indicates the bleaching area used for fluorescence recovery after photobleaching (FRAP). Scale bar, $5 \mu \mathrm{m}$. Time shown in seconds. Right panel: FRAP data points represent means obtained from 28 control cells (siC) and 21 siATIP3-transfected cells (siA), collected from 3 independent experiments. The mean values of double normalized pixel intensities were plotted as a function of time and single exponential curves were fitted $\left(R^{2}>0.99\right)$. Kif2A-GFP fluorescence was recovered to $89 \%$ in control cells and to $86 \%$ in siATIP3-transfected cells. The half-lives of Kif2A-GFP fluorescence recovery were calculated for control $(11.62 \mathrm{~s})$ and siATIP3-transfected cells (11.6 s). Error bars represent standard deviation. Representative immunoblotting data obtained from the indicated total cell extracts used in FRAP experiment is shown below the curves. b Left panel: HeLa cells were transfected with control (siC), ATIP3specific (siA) and/or Dda3-specific (siD) siRNA as indicated and analyzed by immunoblotting with anti-MTUS1 (ATIP3), anti-Kif2A and anti-Dda3 antibodies. Middle panel: Immunofluorescence imaging of HeLa cells transfected as in left panel, then fixed and stained with anti-Kif2A (green) antibodies and DAPI (blue). Scale bar, $10 \mu \mathrm{m}$. Right panel: Quantification of Kif2A fluorescence intensity at the poles as in Fig. $2 \mathrm{~b}$ and quantification of spindle length as in Fig. 1b. Number of poles (for Kif2A staining) and cells (for spindle measurement) analyzed is under brackets. $* * p<0.01$, *** $p<0.001$. c Immunofluorescence imaging of HeLa cells transfected with control (siC) or ATIP3-specific (siA) siRNA, then fixed and stained with anti-Dda3 antibodies (magenta) and DAPI (blue). Scale bar, $10 \mu \mathrm{m}$. Dda3 fluorescence intensity at the poles was measured using ImageJ software. Lower panel: Quantification of maximal Dda3 intensity in metaphase HeLa cells. Number of poles analyzed is under brackets. $* * * p<0.001$. d Immunofluorescence imaging of MCF-7 cells stably expressing GFP or GFP-ATIP3 as indicated. Cells were fixed and stained with anti-GFP (green), anti-Dda3 (magenta) antibodies and DAPI (blue). Scale bar, $10 \mu \mathrm{m}$. Lower panel: Dda3 fluorescence intensity at the poles was analyzed and quantified as in $\mathbf{c}$. Number of poles analyzed is under brackets. $* p<0.05$. e MCF-7 cells were transfected with GFP-ATIP3 or GFP and immunoprecipitation was performed using anti-GFP magnetic beads. Western blots were probed with anti-Dda3 and anti-GFP antibodies. f MCF-7 cells were transfected for $24 \mathrm{~h}$ with GFP-ATIP3 or GFP after transfection for $48 \mathrm{~h}$ with control (siC), Kif2A-specific (siK) or Dda3-specific (siD) siRNA, and immunoprecipitation was performed using anti-GFP magnetic beads. Western blots were probed with anti-Kif2A, antiDda3 and anti-GFP antibodies. Green asterisks indicate cleavage products of GFP-ATIP3. g MCF-7 cells were transfected with GFPATIP3 or GFP and left asynchronous (AS) or synchronized in mitosis (M). Immunoprecipitation was performed using anti-GFP antibodies. Blots were probed with anti-Dda3, anti-Kif2A, anti-GFP and antiPhosphoH3 antibodies. Red asterisks indicate cleavage products of GFP-ATIP3 blot (Fig. 4b). Fluorescence intensity of the phosphorylated (Thr288) active form of the kinase was also reduced upon ATIP3 depletion (Fig. 4b), suggesting that ATIP3 contributes to maintaining an active pool of Aurora A at the poles of the mitotic spindle.

As shown in Fig. 4c, expression of RNAi-resistant GFP-ATIP3 into ATIP3-depleted cells was able to fully restore Aurora A fluorescence intensity at the spindle poles. Importantly, ATIP3-deletion mutants delCN and delCN2, that do not interact with Kif2A, were also able to rescue the phenotype, indicating that ATIP3-mediated control of Aurora targeting to spindle poles is independent of the ATIP3/Kif2A complex.

We next examined the possibility that Aurora kinase A activity may be involved in the ATIP3 phenotype. To investigate whether the short spindle phenotype induced by ATIP3 depletion may be due to decreased Aurora A kinase activity at the poles, we ectopically expressed a GFP-Aurora A construct (GFP-AurA), or kinase-dead mutant (GFP-AurA$\mathrm{KD})$ in ATIP3-silenced cells and analyzed mitotic cells. As shown in Fig. 4d, active GFP-AurA expressed at moderate levels in ATIP3 deficient cells was able to restore a correct mitotic spindle size whereas the kinase dead GFP-AurAKD mutant remained inefficient. Expression of GFP-AurA construct, but not the kinase dead GFP-AurA-KD mutant, also reverted the effects of ATIP3 silencing on Kif2A fluorescence intensities at the poles (Fig. 4e). Together, these results demonstrate that Aurora A kinase activity rescues mitotic spindle defects induced by ATIP3 deficiency, and highlight the central role of Aurora A in the control of metaphase spindle length by ATIP3.

\section{Discussion}

Tight control of mitotic spindle length during metaphase is essential to ensure correct cell division. Results presented here indicate that microtubule-associated protein ATIP3 controls metaphase spindle length by interacting with the microtubule depolymerizing kinesin Kif2A to negatively regulate its association with spindle poles. ATIP3 depletion causes increased Kif2A targeting to the poles, reduced poleward microtubule flux and shorter spindle size. Increased accumulation of Kif2A on spindle poles, and subsequent excessive depolymerization of spindle microtubule minus ends, may disrupt the balance between microtubule polymerization at kinetochores and depolymerization at spindle poles in metaphase, thereby leading to mild changes in poleward microtubule flux. Moderate negative regulation of microtubule flux observed upon ATIP3 depletion may also be due to its negative effect on Aurora kinase A, which was shown to regulate poleward flux via TPX2 and CLASP1 [13].

We show here by fluorescence recovery after photobleaching (FRAP) analyses that the binding of Kif2A on spindle poles is dynamic. Cytosolic pools of GFP-Kif2A were found to exchange in and out of spindle poles with halfrecovery time around $10 \mathrm{~s}$, which is a little faster than for other spindle pole-associated proteins such as NuMA [37]. Of note, ATIP3 silencing has no significant effect on Kif2A turnover at the poles, ruling out the hypothesis that ATIP3 may regulate the dynamic exchange between cytosolic and spindle pole pools of Kif2A proteins. The effect of ATIP3
574

\begin{tabular}{|l|l|l|l|l|}
\hline Journal : Large 18 & Article No : 3614 & Pages : 15 & MS Code : 3614 & Dispatch : 10-8-2020 \\
\hline
\end{tabular}


a

GFP-

GFP ATIP3 GFP ATIP3

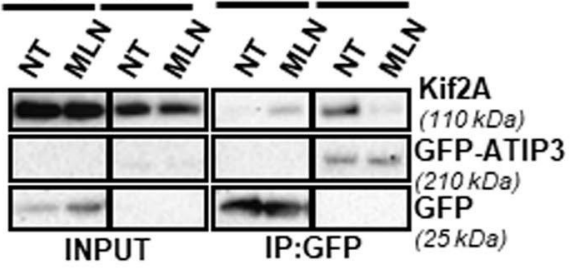

b

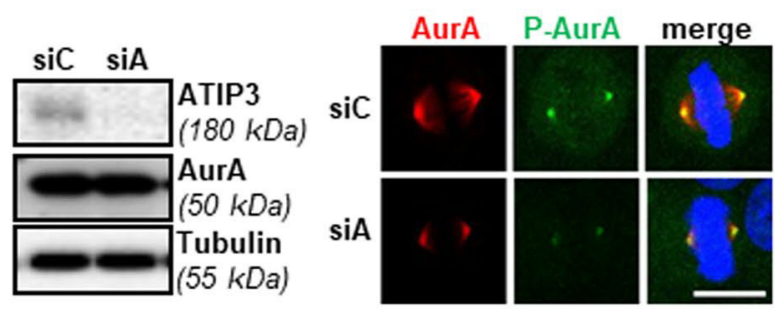

C

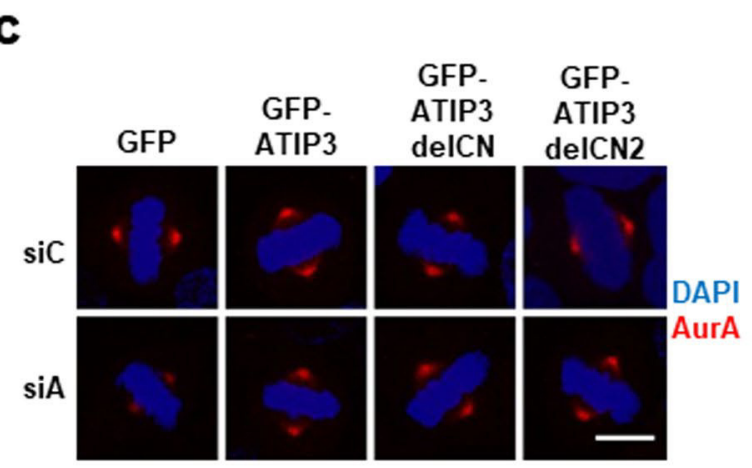

d
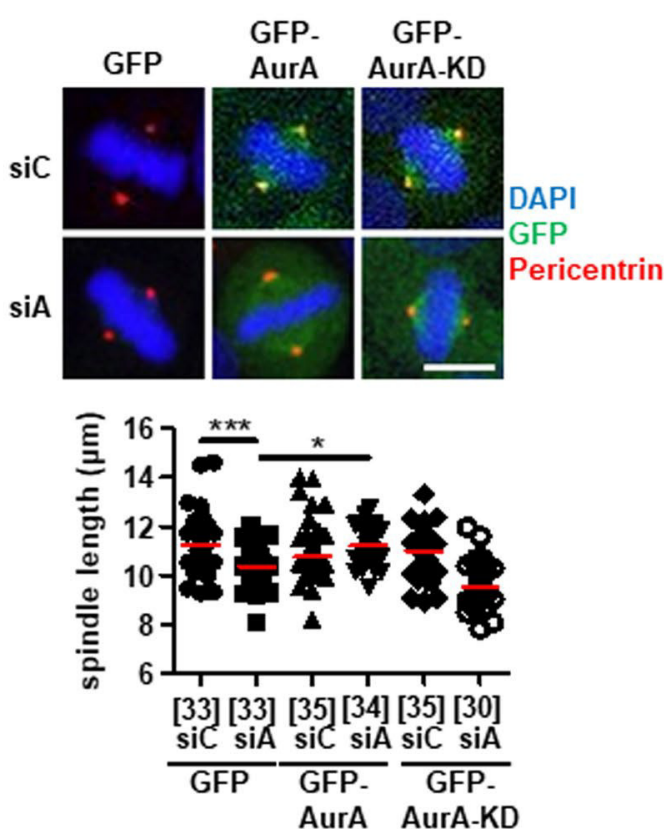

GFP.

GFP.

GFP ATIP3 GFP ATIP3

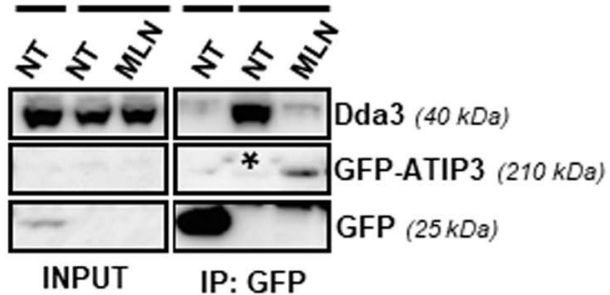

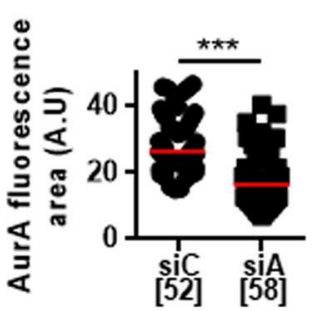
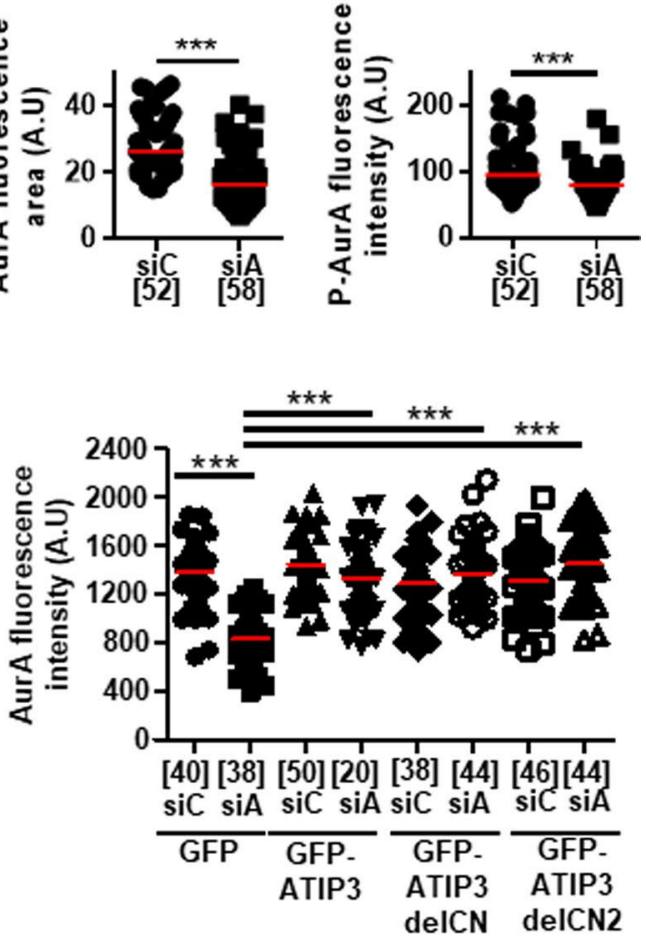

e
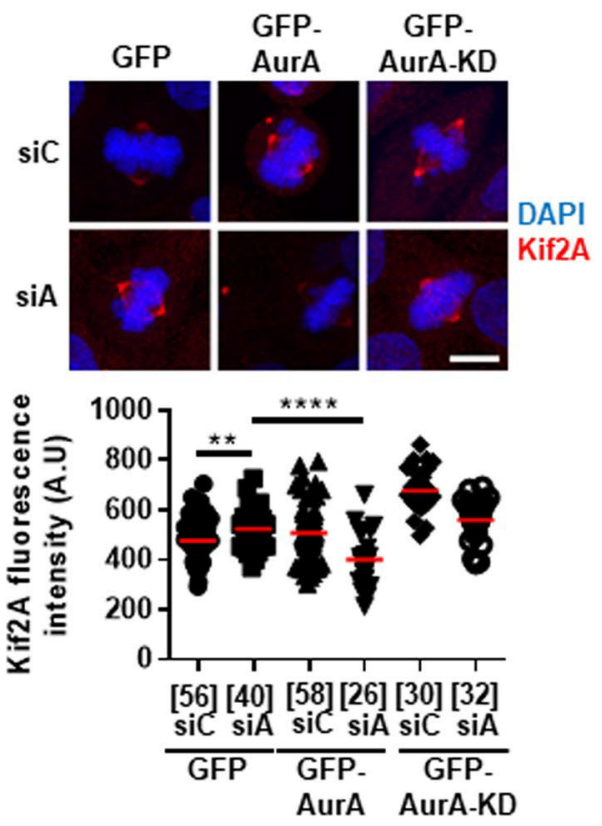
4Fig. 4 Aurora kinase A plays a pivotal role in the ATIP3 phenotype. a MCF-7 cells were transfected with GFP-ATIP3 or GFP, then treated with $500 \mathrm{nM}$ MLN8054 (MLN) (s1100, Selleckchem) or vehicle (NT) for $30 \mathrm{~min}$ at $37^{\circ} \mathrm{C}$, and immunoprecipitation was performed using anti-GFP antibodies. Blots were probed with anti-GFP antibodies and anti-Kif2A (left panel) or anti-Dda3 (right panel) antibodies. A black star indicates weakly detectable immunoprecipitated GFPATIP3. b Left panel: HeLa cells were transfected with control (siC) or ATIP3-specific (siA) siRNA as indicated. Immunoblotting was performed using anti-MTUS1 (ATIP3), anti-Aurora kinase A (AurA) and anti-tubulin antibodies, and indicates that ATIP3-silencing does not modify total expression levels of Aurora A. Middle panel: cells were fixed and stained with anti-Aurora A (AurA, red), anti-phosphoAurora A (P-AurA, green) antibodies and DAPI (blue). Scale bar, $10 \mu \mathrm{m}$. Right panels: Quantification of the area occupied by total Aurora A fluorescence on spindles and poles, measured with ImageJ software. Phospho-Aurora A maximal fluorescence intensity at the poles was measured and quantified as in $\mathbf{b}$. Number of poles analyzed is under brackets. $* * * p<0.001$. c Immunofluorescence imaging of HeLa cells transfected with control (siC) or ATIP3- specific (siA) siRNA for $48 \mathrm{~h}$ and then with GFP, GFP-ATIP3 or deletion mutants GFP-ATIP3delCN and GFP-ATIP3delCN2 for $24 \mathrm{~h}$ as indicated. Cells were fixed and stained with anti-Aurora A (AurA, red) antibodies and DAPI (blue). Scale bar, $10 \mu \mathrm{m}$. Right panel: quantification of Aurora A kinase fluorescence intensity as in $\mathbf{b}$. Number of poles analyzed in under brackets. $* * * p<0.001$. d Immunofluorescence imaging of HeLa cells transfected with control (siC) or ATIP3- specific (siA) siRNA for $48 \mathrm{~h}$ and then with GFP, GFP-AurA or GFP-AurA$\mathrm{KD}$ constructs for $24 \mathrm{~h}$ as indicated. Cells were fixed and stained with anti-GFP (green), anti-pericentrin (red) antibodies and DAPI (blue). Note that all GFP-fusion constructs are expressed at similar levels. Right panel: Quantification of pole-to-pole distance. Number of cells analyzed is under brackets. $* p<0.05, * * * p<0.001$. e Immunofluorescence imaging of HeLa cells transfected as in d, then fixed and stained with anti-Kif2A (red) antibody and DAPI (blue). Right panel: Quantification of Kif2A intensity at poles as in $\mathbf{b}$. Number of poles analyzed is under brackets. $* * p<0.01, * * * p<0.001$

rather involves Dda3, a microtubule-associated protein that binds Kif2A and facilitates its recruitment to the poles [17]. As for Kif2A, ATIP3 depletion increases Dda3 accumulation on the poles. Dda3 silencing in ATIP3-deficient cells rescues the phenotype and restores basal levels of Kif2A fluorescence intensity on the poles as well as a correct size of the spindle. These findings strongly suggest that Dda3 contributes to ATIP3 effects on spindle length by regulating Kif2A recruitment to the poles. While ATIP3 regulates both Kif2a and Dda3 targeting to the poles, silencing of either Kif2A or Dda3 has no effect on ATIP3 localization, suggesting that ATIP3 is upstream of Kif2A/Dda3 and orchestrates the targeting of both proteins to the poles to control spindle length.

ATIP3 interacts with Kif2A and Dda3 in a molecular complex that is increased in mitosis and requires phosphorylation by the mitotic kinase Aurora A. In return, ATIP3 is necessary to maintain an active pool of Aurora A at spindle poles, indicating a positive regulatory loop between Aurora A and ATIP3.

In the absence of ATIP3, Aurora A levels at spindle poles are decreased whereas those of Kif2A and Dda3 are increased. Expression of GFP-Aurora A kinase-but not a kinase-dead mutant - in ATIP3-depleted cells restored basal levels of Kif2A localized at the poles as well as a correct spindle size, confirming the requirement of Aurora kinase activity in the mitotic effects of ATIP3. Together these studies favor a model by which ATIP3 regulates the localization of active Aurora kinase A during mitosis to limit Kif2A recruitment to the poles and control spindle length.

The negative regulation of Kif2A accumulation at spindle poles during metaphase is essential to prevent excessive depolymerization of microtubule minus ends that may lead to mitotic abnormalities. Besides ATIP3, other negative regulators of the Dda3/Kif2A complex have been reported. Mdp3 (microtubule-associated protein 7 domain-containing 3 ) forms a complex with Dda3 and Kif2A in mitosis and counteracts Dda3-mediated Kif2A recruitment to the pole [39]. Another Dda3 partner, the E3 ubiquitin ligase ASB7 (Cullin 5-interacting suppressor of cytokine signaling box 7) was shown to degrade Dda3 through the ubiquitin-proteasome degradation pathway, thereby reducing Kif2A recruitment to the pole [40]. Our data reveal a novel mechanism for negative regulation of Kif2A/Dda3 complex recruitment to the poles, which involves Aurora kinase A and its reciprocal regulation with ATIP3. Of note, ATIP3 weakly interacts with Aurora A and its amino acid sequence presents several potential Aurora A phosphorylation sites, raising the possibility that ATIP3 may also be a substrate of Aurora A. TPX2, a well-known substrate and regulator of Aurora A, also interacts with Kif2A [13] and its phosphorylation by Aurora A contributes to the regulation of poleward microtubule flux and spindle size [13, 22]. Future studies should investigate potential involvement of TPX2 in the ATIP3 phenotype.

In conclusion, results presented here provide the first evidence for the role of Aurora kinase $\mathrm{A}$ in the formation of a molecular complex comprising ATIP3, Kif2A and Dda3, highlighting the pivotal role of ATIP3 and Aurora A kinase cross-regulation on spindle length. This study extends ourA knowledge on the control of the mitotic spindle size in metaphase.

Acknowledgements We thank Dr. Sylvie RODRIGUES-FERREIRA (Inovarion SAS, Paris, France) for helpful discussion. We are grateful to Dr Jamel CHELLY (Institut de Génétique et de Biologie Moléculaire et Cellulaire, Strasbourg, France) for the kind gift of Kif2A-GFP construct and Dr Stephan GELEY (Innsbruck Medical University) for U2OS-mEOS-tubulin cells. We wish to thank Dr Vasily OGRYZKO and Aline VOUILLON (Proteomics Platform of the Gustave Roussy Cancer Campus, Villejuif, France) and Fred LEEUW and Corinne LAPLACE (Plate-forme d'Imagerie et Cytométrie of the Gustave Roussy Cancer Campus, UMS 23/3655, Villejuif, France) for technical assistance and helpful discussion.

Funding This work was supported by the Inserm, the CNRS, the Ligue Nationale Contre le Cancer (Grant no. LNCC94s), the Institut Gustave Roussy, the Labex LERMIT, the GEFLUC association, the Fonds de Dotation Agnès b., the Fondation Janssen Horizon, AG2R

\begin{tabular}{|l|l|l|l|l|}
\hline Journal : Large 18 & Article No : 3614 & Pages : 15 & MS Code : 3614 & Dispatch : 10-8-2020 \\
\hline
\end{tabular}


La Mondiale, the associations Odyssea and Prolific (grants to C.N), by the "Région Ile-de-France" and Fondation pour la Recherche Médicale (grants to D.L.), by grants from Taxe d'apprentissage TA2015 and TA2018 (University Paris-Saclay, France) (grants to A.N) and grants from Lundbeck Foundation (R215-2015-4081) and Danish Cancer Society Research Council (KBVU-R146-A9322) (grants to MB) and Fondation ARC pour la Recherche sur le Cancer.

\section{Compliance with ethical standards}

Conflict of interest The authors declare that they have no conflict of interest.

\section{References}

1. Dumont S, Mitchison TJ (2009) Force and length in the mitotic spindle. Curr Biol 19:R749-R761. https://doi.org/10.1016/j. cub.2009.07.028

2. Goshima G, Scholey JM (2010) Control of mitotic spindle length. Annu Rev Cell Dev Biol 26:21-57. https://doi. org/10.1146/annurev-cellbio-100109-104006

3. Prosser SL, Pelletier L (2017) Mitotic spindle assembly in animal cells: a fine balancing act. Nat Rev Mol Cell Biol 18:187201. https://doi.org/10.1038/nrm.2016.162

4. Mitchison TJ (1989) Polewards microtubule flux in the mitotic spindle: evidence from photoactivation of fluorescence. J Cell Biol 109:637-652

5. Mitchison TJ, Salmon ED (1992) Poleward kinetochore fiber movement occurs during both metaphase and anaphase- $A$ in newt lung cell mitosis. J Cell Biol 119:569-582

6. Ganem NJ, Compton DA (2006) Functional roles of poleward microtubule flux during mitosis. Cell Cycle 5:481-485. https:// doi.org/10.4161/cc.5.5.2519

7. Goshima G, Wollman R, Stuurman N et al (2005) Length control of the metaphase spindle. Curr Biol 15:1979-1988. https:// doi.org/10.1016/j.cub.2005.09.054

8. Tillement V, Remy M-H, Raynaud-Messina B et al (2009) Spindle assembly defects leading to the formation of a monopolar mitotic apparatus. Biol Cell 101:1-11. https://doi.org/10.1042/ BC20070162

9. Young S, Besson S, Welburn JPI (2014) Length-dependent anisotropic scaling of spindle shape. Biol Open 3:1217-1223. https ://doi.org/10.1242/bio.201410363

10. Gaetz J, Kapoor TM (2004) Dynein/dynactin regulate metaphase spindle length by targeting depolymerizing activities to spindle poles. J Cell Biol 166:465-471. https://doi.org/10.1083/ jcb.200404015

11. Ganem NJ, Upton K, Compton DA (2005) Efficient mitosis in human cells lacking poleward microtubule flux. Curr Biol 15:1827-1832. https://doi.org/10.1016/j.cub.2005.08.065

12. Rath U, Rogers GC, Tan D et al (2009) The Drosophila kinesin-13, KLP59D, impacts Pacman- and Flux-based chromosome movement. Mol Biol Cell 20:4696-4705. https://doi. org/10.1091/mbc.e09-07-0557

13. Fu J, Bian M, Xin G et al (2015) TPX2 phosphorylation maintains metaphase spindle length by regulating microtubule flux. $\mathrm{J}$ Cell Biol 210:373-383. https://doi.org/10.1083/jcb.201412109

14. Manning AL, Ganem NJ, Bakhoum SF et al (2007) The kinesin-13 proteins Kif2a, Kif2b, and Kif2c/MCAK have distinct roles during mitosis in human cells. Mol Biol Cell 18:2970 2979. https://doi.org/10.1091/mbc.E07-02-0110
15. Desai A, Verma S, Mitchison TJ, Walczak CE (1999) Kin I kinesins are microtubule-destabilizing enzymes. Cell 96:69-78. https ://doi.org/10.1016/S0092-8674(00)80960-5

16. Cameron LA, Yang G, Cimini D et al (2006) Kinesin 5-independent poleward flux of kinetochore microtubules in PtK1 cells. J Cell Biol 173:173-179. https://doi.org/10.1083/jcb.200601075

17. Jang C-Y, Wong J, Coppinger JA et al (2008) DDA3 recruits microtubule depolymerase Kif2a to spindle poles and controls spindle dynamics and mitotic chromosome movement. J Cell Biol 181:255-267. https://doi.org/10.1083/jcb.200711032

18. Jang C-Y, Coppinger JA, Yates JR, Fang G (2011) Mitotic kinases regulate MT-polymerizing/MT-bundling activity of DDA3. Biochem Biophys Res Commun 408:174-179. https:// doi.org/10.1016/j.bbrc.2011.04.004

19. Jang C-Y, Coppinger JA, Seki A et al (2009) Plk1 and Aurora A regulate the depolymerase activity and the cellular localization of Kif2a. J Cell Sci 122:1334-1341. https://doi.org/10.1242/ jcs.044321

20. Barr AR, Gergely F (2007) Aurora-A: the maker and breaker of spindle poles. J Cell Sci 120:2987-2996. https://doi.org/10.1242/ jcs.013136

21. Magnaghi-Jaulin L, Eot-Houllier G, Gallaud E, Giet R (2019) Aurora A protein kinase: to the centrosome and beyond. Biomolecules. https://doi.org/10.3390/biom9010028

22. Bird AW, Hyman AA (2008) Building a spindle of the correct length in human cells requires the interaction between TPX2 and Aurora A. J Cell Biol 182:289-300. https://doi.org/10.1083/ jcb.200802005

23. Rodrigues-Ferreira S, Di Tommaso A, Dimitrov A et al (2009) 8p22 MTUS1 gene product ATIP3 is a novel anti-mitotic protein underexpressed in invasive breast carcinoma of poor prognosis. PLoS ONE 4:e7239. https://doi.org/10.1371/journal.pone.00072 39

24. Molina A, Velot L, Ghouinem L et al (2013) ATIP3, a novel prognostic marker of breast cancer patient survival, limits cancer cell migration and slows metastatic progression by regulating microtubule dynamics. Cancer Res 73:2905-2915. https://doi. org/10.1158/0008-5472.CAN-12-3565

25. Velot L, Molina A, Rodrigues-Ferreira S et al (2015) Negative regulation of EB1 turnover at microtubule plus ends by interaction with microtubule-associated protein ATIP3. Oncotarget 6:43557-43570. https://doi.org/10.18632/oncotarget.6196

26. Nehlig A, Molina A, Rodrigues-Ferreira $S$ et al (2017) Regulation of end-binding protein EB1 in the control of microtubule dynamics. Cell Mol Life Sci 74:2381-2393. https://doi.org/10.1007/ s00018-017-2476-2

27. Rodrigues-Ferreira S, Nehlig A, Moindjie H et al (2019) Improving breast cancer sensitivity to paclitaxel by increasing aneuploidy. Proc Natl Acad Sci USA 116:23691-23697. https://doi. org/10.1073/pnas.1910824116

28. Meraldi P, Honda R, Nigg EA (2002) Aurora-A overexpression reveals tetraploidization as a major route to centrosome amplification in $\mathrm{p}^{-1-}$ cells. EMBO J 21:483-492

29. Rannou Y, Troadec M-B, Petretti C et al (2008) Localization of aurora A and aurora B kinases during interphase: role of the N-terminal domain. Cell Cycle 7:3012-3020. https://doi.org/10.4161/ cc.7.19.6718

30. Courtheoux T, Diallo A, Damodaran AP et al (2018) Aurora A kinase activity is required to maintain an active spindle assembly checkpoint during prometaphase. J Cell Sci 131:jcs191353. https ://doi.org/10.1242/jcs.191353

31. Poullet P, Carpentier S, Barillot E (2007) myProMS, a web server for management and validation of mass spectrometry-based proteomic data. Proteomics 7:2553-2556. https://doi.org/10.1002/ pmic. 200600784

\begin{tabular}{|l|l|l|l|l|}
\hline Journal : Large 18 & Article No: 3614 & Pages : 15 & MS Code : 3614 & Dispatch : 10-8-2020 \\
\hline
\end{tabular}


32. Valot B, Langella O, Nano E, Zivy M (2011) MassChroQ: a versatile tool for mass spectrometry quantification. Proteomics 11:3572-3577. https://doi.org/10.1002/pmic.201100120

33. Kowal J, Arras G, Colombo M et al (2016) Proteomic comparison defines novel markers to characterize heterogeneous populations of extracellular vesicle subtypes. Proc Natl Acad Sci USA 113:E968-E977. https://doi.org/10.1073/pnas.1521230113

34. Vizcaíno JA, Csordas A, del-Toro N et al (2016) 2016 update of the PRIDE database and its related tools. Nucleic Acids Res 44:D447-D456. https://doi.org/10.1093/nar/gkv1145

35. Wandke C, Barisic M, Sigl R et al (2012) Human chromokinesins promote chromosome congression and spindle microtubule dynamics during mitosis. J Cell Biol 198:847-863. https://doi. org/10.1083/jcb.201110060

36. Pereira AJ, Maiato H (2010) Improved kymography tools and its applications to mitosis. Methods San Diego Calif 51:214-219. https://doi.org/10.1016/j.ymeth.2010.01.016

37. Stenoien DL, Sen S, Mancini MA, Brinkley BR (2003) Dynamic association of a tumor amplified kinase, Aurora-A, with the centrosome and mitotic spindle. Cell Motil Cytoskelet 55:134146. https://doi.org/10.1002/cm.10120

38. Jang C-Y, Coppinger JA, Yates JR, Fang G (2010) Phospho-regulation of DDA3 function in mitosis. Biochem Biophys Res Commun 393:259-263. https://doi.org/10.1016/j.bbrc.2010.01.115

39. Kwon HJ, Park JE, Song H, Jang C-Y (2016) DDA3 and Mdp3 modulate Kif2a recruitment onto the mitotic spindle to control minus-end spindle dynamics. J Cell Sci 129:2719-2725. https:// doi.org/10.1242/jcs.180109

40. Uematsu K, Okumura F, Tonogai S et al (2016) ASB7 regulates spindle dynamics and genome integrity by targeting DDA3 for proteasomal degradation. J Cell Biol 215:95-106. https://doi. org/10.1083/jcb.201603062

Publisher's Note Springer Nature remains neutral with regard to jurisdictional claims in published maps and institutional affiliations. 


\begin{tabular}{|ll|}
\hline Journal: & $\mathbf{1 8}$ \\
Article: & $\mathbf{3 6 1 4}$ \\
\hline
\end{tabular}

\section{Author Query Form}

\section{Please ensure you fill out your response to the queries raised below and return this form along with your corrections}

Dear Author

During the process of typesetting your article, the following queries have arisen. Please check your typeset proof carefully against the queries listed below and mark the necessary changes either directly on the proof/online grid or in the 'Author's response' area provided below

\begin{tabular}{|l|l|l|}
\hline Query & Details Required & Author's Response \\
\hline AQ1 & $\begin{array}{l}\text { Author details: Kindly check and confirm whether the corresponding author is } \\
\text { correctly identified. }\end{array}$ & \\
\hline AQ2 & Kindly check and confirm the organization name in affiliation 1. & \\
\hline AQ3 & Please confirm all the section headings are correctly identified. & \\
\hline AQ4 & $\begin{array}{l}\text { Kindly check and confirm the inserted grant number (LNCC94) and funder name } \\
\text { 'Fondation ARC pour la Recherche sur le Cancer' in funding statement. }\end{array}$ & \\
\hline AQ5 & Kindly check and confirm the page number for the reference [30]. & \\
\hline
\end{tabular}

